\section{Consumo abusivo e dependência de álcool em população adulta no Estado de São Paulo, Brasil}

\section{Alcohol abuse and dependence in adults in the State of São Paulo, Brazil}

Vanessa Valente Guimarães'

Alex Antônio Florindo'

Sheila Rizzato Stopa'

Chester Luiz Galvão César"

Marilisa Berti de Azevedo Barros"'I

Luana Carandinalv

Moisés Goldbaum ${ }^{v}$

'Escola de Artes, Ciências e Humanidades da Universidade de São Paulo (USPLeste)

"Departamento de Epidemiologia da Faculdade de Saúde Pública da USP

"'Departamento de Medicina Preventiva e Social, Faculdade de Ciências Médicas da Universidade Estadual de Campinas (UNICAMP)

IVDepartamento de Saúde Pública da Faculdade de Medicina de Botucatu da Universidade Estadual Paulista (UNESP)

${ }^{\vee}$ Departamento de Medicina Preventiva da Faculdade de Medicina da USP

Estudo financiado pela Fundação de Amparo à Pesquisa do Estado de São Paulo-FAPESP (Processo n० 98/14099-7) e Secretaria da Saúde do Estado de São Paulo.

Correspondência: Vanessa Valente Guimarães. Escola de Artes, Ciências e Humanidades da Universidade de São Paulo - USP-Leste. Rua Arlindo Béttio 1.000 - Ermelino Matarazzo - São Paulo, SP CEP 03828-000. vanessavalente@usp.br

\section{Resumo}

Objetivo: Descrever as prevalências de consumo abusivo e dependência de álcool em população adulta de 20 a 59 anos no Estado de São Paulo, e suas associações com variáveis demográficas e socioeconômicas. Métodos: Inquérito domiciliar do tipo transversal (ISA-SP), em quatro áreas do Estado de São Paulo: a) Região Sudoeste da Grande São Paulo, constituída pelos Municípios de Taboão da Serra, Itapecerica da Serra e Embu; b) Distrito do Butantã, no Município de São Paulo; c) Município de Campinas e; d) Município de Botucatu. Foi considerado consumo abusivo de álcool a ingestão em dia típico de 30 gramas ou mais de etanol para os homens, e 24 gramas ou mais para as mulheres. A dependência de álcool foi caracterizada pelo questionário CAGE. Análises bivariadas e multivariadas dos dados foram realizadas a partir de Modelos de Regressão de Poisson. Todas as análises foram estratificadas por sexo. Resultados: Em 1.646 adultos entrevistados, a prevalência de consumo abusivo de álcool foi de 52,9\% no sexo masculino e $26,8 \%$ no sexo feminino. Quanto à dependência de álcool, foram observadas duas ou mais respostas positivas no teste CAGE em $14,8 \%$ dos homens e em $5,4 \%$ das mulheres que relataram consumir álcool. Isto corresponde a uma prevalência populacional de dependência de $10,4 \%$ nos homens e 2,6\% nas mulheres. O consumo abusivo de álcool no sexo masculino apresentou associação inversa à faixa etária e associação direta à escolaridade e ao tabagismo. No sexo feminino, observou-se associação direta do consumo abusivo de álcool com a escolaridade e o tabagismo, e com as situações conjugais sem companheiro. A dependência de álcool no sexo masculino associou-se a não exercer atividade de trabalho e à baixa escolaridade. No sexo feminino não houve associação do CAGE com nenhuma das variáveis estudadas. Conclusões: Pela alta prevalência de consumidores e dependentes, é essencial a identificação dos segmentos sociodemográficos mais vulneráveis ao consumo abusivo e dependência de álcool. As associações entre a dependência/abuso e não estar exercendo atividade de trabalho, no sexo masculino, $\mathrm{e}$ a maior prevalência em mulheres de escolaridade universitária, sugerem componentes para programas de intervenção e controle.

Palavras-chave: Abuso de álcool. Dependência de álcool. Saúde do adulto. Inquéritos de saúde. 


\section{Abstract}

Objetive: To investigate alcohol abuse and dependence in adults aged 20-59 years, in the State of São Paulo, Brazil, according to demographic and socio-economic characteristics. Methods: Cross-sectional household survey carried out in four areas of the State of São Paulo, Brazil. The CAGE questionnaire was used to investigate alcohol dependence. Alcohol abuse was defined as daily consumption of at least 30 grams of alcohol for men and 24 grams for women. Bivariate and multivariate Poisson regression analyses were performed to detect associations and high-risk groups. All analyses were stratified by gender. Results: 1,646 adults were interviewed. The prevalence of alcohol abuse was $52.9 \%$ among men and $26.8 \%$ in women. With a CAGE cutoff point $\geq 2$, alcohol dependence was found in $14.8 \%$ of male drinkers and $5.4 \%$ of female drinkers. These proportions correspond to a population prevalence of alcohol addiction of $10.4 \%$ in men and $2.6 \%$ in women. With regard to alcohol abuse, in men, it was negatively associated with age and directly associated with schooling and smoking. In women, alcohol abuse was also associated with schooling and smoking, and with living without a partner. In men, a significant association was found between alcohol dependence and lower schooling levels. Unemployment was also significantly associated with alcohol dependence in men. No overall association was found in alcohol dependence in women. Conclusions: Our data revealed high prevalences of alcohol abuse and dependence. The association of alcohol abuse with higher schooling and the finding of alcohol dependence among unemployed men suggest elements for intervention and control policies.

Keywords: Alcohol abuse. Alcohol dependence. Adults. Health surveys.

\section{Introdução}

O consumo abusivo de álcool é reconhecido como um importante problema de saúde pública em todo o mundo ${ }^{1}$. $\mathrm{O}$ abuso/ dependência de álcool estão associados a múltiplas consequências adversas para a saúde, como doenças cardíacas e cerebrovasculares ${ }^{1}$, eventos fatais e transtornos psiquiátricos ${ }^{2}$, traumas $^{3}$, violência doméstica ${ }^{4}$, quedas $^{5}$, várias neoplasias ${ }^{6}$, doenças sexualmente transmissíveis ${ }^{7}$, cirrose hepática ${ }^{8}$, dentre outros. No Brasil, estudo conduzido em 1999, no Estado de São Paulo, apontou 6,6\% da população entre 12 e 65 anos de idade com dependência de álcool ${ }^{9}$. Dois anos depois, a mesma população foi pesquisada e foi constatado aumento estatisticamente significativo para $9,4 \%$ de dependentes ${ }^{10}$.

Uma avaliação das principais causas de morte nos Estados Unidos da América no ano 2000 concluiu que $18,1 \%$ do total de óbitos naquele país eram atribuídos ao uso de tabaco, $16,6 \%$ a dieta inadequada $\mathrm{e}$ inatividade física, e 3,5\% ao consumo de álcool ${ }^{11}$. Fatores modificáveis relacionados ao estilo de vida respondem pelas quatro principais causas de morte naquele país. Assim, a mensuração e o monitoramento dos padrões de ingestão alcoólica, com detecção dos segmentos mais vulneráveis, é tarefa essencial para planejamento de estratégias e ações para controle do consumo abusivo, prevenção de doenças crônicas e promoção da saúde.

O objetivo do presente estudo é descrever as prevalências de consumo abusivo e dependência de álcool em população adulta em quatro áreas do Estado de São Paulo, e explorar suas associações com variáveis demográficas e socioeconômicas.

\section{Métodos}

Trata-se de estudo transversal, de base populacional, que utilizou dados da pesquisa Inquérito de Saúde no Estado de São Paulo (ISA-SP), cujas informações foram coletadas nos anos de 2001 e 2002 em quatro áreas do Estado de São Paulo: a) Região Sudoeste da 
Grande São Paulo, constituída pelos municípios de Taboão da Serra, Embu e Itapecerica da Serra; b) Distrito do Butantã, no Município de São Paulo; c) Município de Campinas e; d) Município de Botucatu. A pesquisa foi realizada por três universidades públicas do Estado (Universidade de São Paulo - USP, Universidade Estadual Paulista - UNESP e Universidade Estadual de Campinas - UNICAMP) e contou com a parceria da Secretaria de Saúde do Estado de São Paulo.

A amostragem foi probabilística, por conglomerados, em dois estágios: setor censitário e domicílio. As unidades primárias de amostragem (setores censitários) foram sorteadas com probabilidade proporcional ao tamanho, a partir dos dados da Contagem Populacional do IBGE de 1996. Os domicílios foram sorteados com probabilidade inversamente proporcional ao tamanho do setor, a partir de listagens feitas em visitas aos setores sorteados.

O projeto ISA-SP estudou grupos temáticos, sendo que para cada um deles foram formuladas questões, em sua maioria fechadas. Os principais conjuntos temáticos são: condições de vida, morbidade referida, doenças crônicas, utilização de serviços de saúde e estilo de vida. Este estudo foi aprovado pelo Comitê de Ética da Faculdade de Saúde Pública da USP.

A população do presente estudo incluiu 1.646 indivíduos adultos, não-institucionalizados, de ambos os sexos, com idade entre 20 e 59 anos, residentes em área urbana dos municípios pesquisados. Os dados foram obtidos por meio de questionários aplicados por entrevistadores treinados, respondidos diretamente pelos moradores adultos sorteados.

As variáveis dependentes deste estudo foram consumo abusivo de álcool e dependência de álcool. O ponto de corte para definir consumo abusivo foi de 30 gramas/ dia de etanol ou mais para o sexo masculino, e 24 gramas/dia ou mais para o sexo feminino $^{12}$. A dependência de álcool foi avaliada por meio do CAGE, questionário utilizado para detectar possível dependência de álcool em estudos clínicos e populacionais.
É composto de quatro perguntas (Cut down

- reduzir a ingesta / Annoyed by criticism - irritado / Guilty - culpado / Eye-opener identificação de ressaca) e considera como caso suspeito de dependência de álcool, ou "bebedor-problema", alguém que responda afirmativamente a duas ou mais perguntas. No Brasil, sua validação foi feita por Masur \& Monteiro $^{13}$, em 1983, observando-se sensibilidade de $88,0 \%$ e especificidade de $83,0 \%$. No ISA-SP, foi considerado indicativo de dependência quando apresentou duas ou mais respostas SIM.

As variáveis independentes estudadas foram sexo, faixa etária, situação conjugal, escolaridade, renda familiar mensal em salários-mínimos per capita, exercer atividade de trabalho e tabagismo.

Na análise geral dos dados, os programas de processamento e análise incorporaram os fatores de ponderação da amostra, baseados no sexo e idade dos entrevistados e na fração amostral do setor censitário, a partir do percentual de chefes de família com nível universitário. Os cálculos para definições dos pesos utilizaram as informações do Censo IBGE de 2000.

Para avaliar o efeito das variáveis demográficas e socioeconômicas sobre as variáveis do consumo abusivo e dependência de álcool, optou-se por realizar Regressão de Poisson com estimação robusta - modelagem que proporciona melhor ajuste e estima diretamente as razões de prevalências e seus intervalos de confiança ${ }^{14-16}$.

Para a análise de Regressão de Poisson simples, todas as variáveis foram avaliadas em categorias e transformadas em variáveis indicadoras, permitindo a comparação das razões de prevalência entre as categorias de cada variável analisada. As variáveis que obtiveram $p$ menor que 0,20 na análise de Regressão de Poisson simples foram incluídas nos modelos múltiplos. Optamos pela modelagem stepwise forward, ou seja, do modelo mais simples para o mais complexo. As variáveis que permaneceram significativas foram mantidas nos modelos múltiplos finais. Tendências lineares foram exploradas para variáveis categóricas ordinais. 
Foram utilizados os softwares SPSS (Statistical Package for the Social Sciences), módulo Complex Samples, versão 15.0 e Stata, módulo Survey, versão 9.0. Todas as análises foram estratificadas por sexo e em todas utilizou-se o nível de significância de 5\%.

\section{Resultados}

A amostra total de adultos obtida do banco de dados do ISA-SP para as quatro áreas de estudo foi de 1.646 indivíduos entrevistados, sendo 826 do sexo masculino e 820 do sexo feminino. Após ponderação, estes valores correspondem a $48,0 \%$ (IC
95\% 45,0-51,0) de entrevistados do sexo masculino e $52,0 \%$ (IC $95 \%$ 49,0-55,0) do sexo feminino.

Quanto à faixa etária observamos, em nossa amostra, predomínio de indivíduos na faixa $20-29$ anos (33,8\%). As faixas etárias 30-39 anos, 40-49 anos e 50-59 anos apresentaram, respectivamente, percentuais de $25,1 \%, 24,1 \%$ e $17,0 \%$. Esta diferença na estrutura etária é estatisticamente significativa $(\mathrm{p}<0,001)$.

A Tabela 1 apresenta as características demográficas e socioeconômicas segundo sexo da população adulta nas quatro áreas. No sexo masculino, observamos que $66,2 \%$ dos

Tabela 1 - Características demográficas e socioeconômicas segundo sexo em população adulta no Estado de São Paulo.

Table 1 - Comparison of demographic and socioeconomic characteristics of the study sample by sex, State of São Paulo, Brazil

\begin{tabular}{|c|c|c|c|c|}
\hline \multirow[t]{2}{*}{ Variável } & \multicolumn{2}{|c|}{ Masculino } & \multicolumn{2}{|c|}{ Feminino } \\
\hline & $\mathrm{n}^{1}$ & $\%^{2}$ & $\mathrm{n}^{1}$ & $\%^{2}$ \\
\hline \multicolumn{5}{|l|}{ Faixa etária } \\
\hline 20-29 anos & 279 & 34,0 & 274 & 33,5 \\
\hline 30-39 anos & 205 & 22,6 & 219 & 27,5 \\
\hline 40-49 anos & 198 & 24,7 & 187 & 23,5 \\
\hline 50-59 anos & 144 & 18,7 & 139 & 15,5 \\
\hline \multicolumn{5}{|l|}{ Situação conjugal } \\
\hline Com companheiro & 550 & 66,2 & 491 & 60,3 \\
\hline Sem companheiro & 273 & 33,8 & 329 & 39,7 \\
\hline \multicolumn{5}{|l|}{ Escolaridade } \\
\hline$<3$ anos & 91 & 12,7 & 117 & 13,8 \\
\hline 4-7 anos & 240 & 29,8 & 221 & 25,0 \\
\hline 8-11 anos & 344 & 40,3 & 320 & 40,5 \\
\hline$\geq 12$ anos & 150 & 17,2 & 162 & 20,7 \\
\hline \multicolumn{5}{|c|}{ Exerce atividade de trabalho } \\
\hline Sim & 676 & 80,9 & 507 & 62,6 \\
\hline Não & 150 & 19,1 & 313 & 37,4 \\
\hline \multicolumn{5}{|c|}{$\begin{array}{l}\text { Renda familiar mensal (salários-mínimos } \\
\text { per capita) }\end{array}$} \\
\hline$<0,5$ & 65 & 8,3 & 87 & 9,0 \\
\hline $0,5-1,49$ & 237 & 29,3 & 243 & 31,2 \\
\hline $1,5-2,9$ & 225 & 28,7 & 209 & 28,2 \\
\hline$\geq 3$ & 255 & 33,6 & 235 & 31,6 \\
\hline
\end{tabular}

'Números absolutos na amostra não-ponderada. 'Unweighted number of observations.

${ }^{2}$ Porcentagens na amostra ponderada. ${ }^{2}$ Weighted sample percentages. 
entrevistados eram casados (com companheira), 40,3\% com escolaridade entre 8-11 anos, e 80,9\% exerciam atividade remunerada de trabalho. Dentre as mulheres, $60,3 \%$ viviam com companheiro, $40,5 \%$ tinham entre $8-11$ anos de escolaridade e $62,6 \%$ exerciam atividade remunerada de trabalho.

Para a maioria das variáveis não houve diferenças significativas entre os sexos feminino e masculino. Houve diferença apenas para as variáveis situação conjugal $(p=0,043)$ e exerce atividade de trabalho $(\mathrm{p}<0,001)$.

A Tabela 2 apresenta as prevalências das variáveis relacionadas ao consumo abusivo, frequência de ingestão e dependência de álcool na população de 20 a 59 anos de idade nas quatro áreas de estudo segundo sexo.

Quanto ao tabagismo, 60,0\% das mulheres relataram nunca ter fumado. No sexo masculino esta freqüência foi de $51,4 \%$. Esta diferença entre os sexos é estatisticamente significativa $(p=0,004)$.
Houve grande diferença entre os sexos quanto à frequência de ingestão de bebida alcoólica $(\mathrm{p}<0,001)$ e prevalência do consumo abusivo de álcool $(\mathrm{p}<0,001)$. Apenas $6,9 \%$ das mulheres referiram ingerir álcool em frequência de duas a três vezes por semana ou mais, enquanto que $32,5 \%$ dos homens adultos relataram beber com esta frequência. Dentre os que referiram ingerir álcool, houve maior percentual de homens com CAGE positivo $(\geq 2)$.

A Tabela 3 apresenta os dados de consumo abusivo de álcool no sexo masculino, onde observamos associação inversa com a faixa etária e associação direta com a escolaridade. No sexo feminino, observou-se associação direta do consumo abusivo de álcool com a escolaridade (Tabela 4).

Ao avaliar o questionário CAGE, indicativo de dependência de álcool, observou-se maior prevalência de CAGE positivo nos homens com menor escolaridade e nos

Tabela 2 - Prevalência de tabagismo, consumo abusivo, dependência e frequência de ingestão de álcool segundo sexo em população adulta no Estado de São Paulo.

Table 2 - Comparison of smoking, alcohol abuse, alcohol dependence, and frequency of alcohol consumption by sex, State of São Paulo, Brazil

\begin{tabular}{lcccc}
\hline Variável & \multicolumn{2}{c}{ Masculino } & \multicolumn{2}{c}{ Feminino } \\
\cline { 2 - 5 } & $\mathrm{n}^{1}$ & $\%^{2}$ & $\mathrm{n}^{1}$ & $\%^{2}$ \\
\hline Tabagismo & & & & \\
$\quad$ Nunca fumou & 403 & 51,4 & 489 & 59,7 \\
$\quad$ Ex-tabagista & 186 & 22,0 & 130 & 15,2 \\
$\quad$ Fuma atualmente & 235 & 26,6 & 201 & 25,1 \\
Consumo abusivo de álcool* & & & & \\
$\quad$ Sim & 442 & 52,9 & 217 & 26,8 \\
$\quad$ Não & 384 & 47,1 & 603 & 73,2 \\
Frequência de ingestão de álcool & & & & \\
$\quad$ Até 1 a 2 vezes/mês & 415 & 47,6 & 667 & 79,0 \\
$\quad$ 1 vez/semana & 153 & 19,9 & 101 & 14,1 \\
$\quad$ a 3 vezes/semana ou mais & 258 & 32,5 & 52 & 6,9 \\
CAGE** & & & & \\
$\quad<2$ & 483 & 85,2 & 351 & 94,6 \\
$\quad 22$ & 82 & 14,8 & 21 & 5,4 \\
\hline
\end{tabular}

'Números absolutos na amostra não-ponderada. 'Unweighted number of observations.

${ }^{2}$ Porcentagens na amostra ponderada. ${ }^{2}$ Weighted sample percentages.

*Caracterizado como ingestão de álcool em dia típico de $30 \mathrm{~g}$ ou mais para homens e $24 \mathrm{~g}$ ou mais para mulheres. *Daily consumption of at least 30 grams of alcohol for men and 24 grams for women.

**Indicador de dependência de álcool. ${ }^{* *}$ Alcohol dependence screening. 
Tabela 3 - Consumo abusivo de álcool' (\%) segundo variáveis demográficas e socioeconômicas em população adulta do sexo masculino, Estado de São Paulo.

Table 3 - Alcohol abuse'(\%) according to demographic and socioeconomic variables in male adults, State of São Paulo, Brazil

\begin{tabular}{|c|c|c|c|c|c|}
\hline \multirow[t]{2}{*}{ Variável } & \multirow[t]{2}{*}{$\%^{1}$} & \multicolumn{2}{|c|}{ Razão de Prevalências } & \multicolumn{2}{|c|}{ Razão de Prevalências } \\
\hline & & Bruta & IC 95\% & Ajustada $^{2}$ & IC 95\% \\
\hline \multicolumn{6}{|l|}{ Faixa etária } \\
\hline 20-29 anos & 55,6 & 1,00 & 1,00 & & \\
\hline 30-39 anos & 56,6 & 1,01 & $0,83-1,24$ & 0,98 & $0,80-1,19$ \\
\hline 40-49 anos & 53,9 & 0,97 & $0,78-1,19$ & 0,95 & $0,77-1,17$ \\
\hline \multirow[t]{2}{*}{ 50-59 anos } & 42,4 & 0,76 & $0,58-1,00$ & 0,73 & $0,56-0,96$ \\
\hline & & \multicolumn{2}{|c|}{$p$ de tendência ${ }^{3}=0,209$} & \multicolumn{2}{|c|}{$p$ de tendência ${ }^{3}=0,142$} \\
\hline \multicolumn{6}{|l|}{ Situação conjugal } \\
\hline Com companheira & 52,8 & 1,00 & 1,00 & & \\
\hline Sem companheira & 53,0 & 1,00 & $0,84-1,19$ & 0,96 & $0,80-1,16$ \\
\hline \multicolumn{6}{|l|}{ Tabagismo } \\
\hline Nunca & 43,9 & 1,00 & 1,00 & & \\
\hline Ex-tabagista & 57,1 & 1,30 & $1,05-1,60$ & 1,39 & $1,12-1,71$ \\
\hline \multirow[t]{2}{*}{ Fumo atual } & 66,9 & 1,52 & $1,27-1,82$ & 1,59 & $1,34-1,90$ \\
\hline & & \multicolumn{2}{|c|}{$\mathrm{p}$ de tendência ${ }^{3}=0,000$} & \multicolumn{2}{|c|}{$p$ de tendência ${ }^{3}=\mathbf{0 , 0 0 0}$} \\
\hline \multicolumn{6}{|l|}{ Escolaridade } \\
\hline Até 3 anos & 37,8 & 1,00 & 1,00 & & \\
\hline 4-7 anos & 62,1 & 1,64 & $1,20-2,24$ & 1,64 & $1,05-1,94$ \\
\hline 8-11 anos & 51,9 & 1,37 & $0,99-1,88$ & 1,43 & $1,03-1,97$ \\
\hline \multirow[t]{2}{*}{$\geq 12$ anos } & 51,3 & 1,35 & $0,95-1,93$ & 1,42 & $1,01-2,03$ \\
\hline & & \multicolumn{2}{|c|}{$\mathrm{p}$ de tendência ${ }^{3}=0,009$} & \multicolumn{2}{|c|}{$\mathrm{p}$ de tendência ${ }^{3}=\mathbf{0 , 0 1 3}$} \\
\hline
\end{tabular}

'Consumo abusivo de álcool definido como ingestão de álcool em dia típico de $30 \mathrm{~g}$ ou mais para homens e $24 \mathrm{~g}$ ou mais para mulheres. Porcentagens na amostra ponderada. ' Alcohol abuse defined as daily consumption of at least 30 grams of alcohol for men and 24 grams for women. Weighted sample percentages. ${ }^{2}$ Regressão de Poisson ajustada para variáveis apresentadas na tabela. ${ }^{2}$ Poisson Regression controlling for variables presented in the table.

${ }^{3}$ Valor de $\mathrm{p}$ para teste de tendência linear. ${ }^{3}$ Trend test.

que não exercem atividade remunerada de trabalho (Tabela 5). No sexo feminino não houve associação do CAGE com nenhuma das variáveis estudadas.

\section{Discussão}

O levantamento de dados para a análise de variáveis relacionadas ao consumo de álcool, frequência, abuso e dependência, tem sido objeto de muitos estudos, com intuito de determinar seu impacto social e morbimortalidade geral. Existem duas estratégias frequentemente utilizadas para determinar o consumo de álcool em uma população: as estimativas per capita e os dados derivados de inquéritos populacionais.
Informações de base populacional apresentam o diferencial de permitir análises sociodemográficas entre grupos específicos numa dada população ${ }^{17}$.

No presente estudo, observou-se elevada prevalência de consumo abusivo de álcool, fortemente associado ao tabagismo e inversamente proporcional à faixa etária em ambos os sexos. Para o sexo masculino, destaca-se o aumento do consumo abusivo com o incremento da escolaridade. Nas mulheres, o consumo abusivo foi associado à escolaridade universitária e também às situações conjugais solteira, separada, divorciada e viúva, ou seja, sem companheiro. A dependência de álcool, avaliada por meio do questionário CAGE $(\geq 2)$, associou-se 
Tabela 4 - Consumo abusivo de álcool' (\%) segundo variáveis demográficas e socioeconômicas em população adulta do sexo feminino, Estado de São Paulo.

Table 4 - Alcohol abuse' (\%) according to demographic and socioeconomic variables in female adults, State of São Paulo, Brazil

\begin{tabular}{|c|c|c|c|c|c|}
\hline \multirow[t]{2}{*}{ Variável } & \multirow[t]{2}{*}{$\%^{1}$} & \multicolumn{2}{|c|}{ Razão de Prevalências } & \multicolumn{2}{|c|}{ Razão de Prevalências } \\
\hline & & Bruta & IC 95\% & Ajustada $^{2}$ & IC 95\% \\
\hline \multicolumn{6}{|l|}{ Faixa etária } \\
\hline 20-29 anos & 28,4 & 1,00 & & 1,00 & \\
\hline 30-39 anos & 27,1 & 0,95 & $0,67-1,36$ & 0,84 & $0,58-1,20$ \\
\hline 40-49 anos & 28,6 & 1,01 & $0,70-1,45$ & 0,85 & $0,57-1,26$ \\
\hline \multirow[t]{2}{*}{$50-59$ anos } & 20,5 & 0,72 & $0,44-1,19$ & 0,58 & $0,35-0,95$ \\
\hline & & \multicolumn{2}{|c|}{$\mathrm{p}$ de tendência ${ }^{3}=0,603$} & \multicolumn{2}{|c|}{$p$ de tendência ${ }^{3}=0,197$} \\
\hline \multicolumn{6}{|l|}{ Situação conjugal } \\
\hline Com companheiro & 22,4 & 1,00 & & 1,00 & \\
\hline Sem companheiro & 33,5 & 1,50 & $1,13-1,98$ & 1,33 & $1,01-1,74$ \\
\hline \multicolumn{6}{|l|}{ Tabagismo } \\
\hline Nunca & 17,6 & 1,00 & & 1,00 & \\
\hline Ex-tabagista & 29,9 & 1,69 & $1,12-2,55$ & 1,91 & $1,26-2,89$ \\
\hline \multirow[t]{2}{*}{ Fumo atual } & 46,9 & 2,66 & $1,96-3,60$ & 2,92 & $2,12-4,00$ \\
\hline & & \multicolumn{2}{|c|}{$p$ de tendência ${ }^{3}=0,000$} & \multicolumn{2}{|c|}{$p$ de tendência ${ }^{3}=\mathbf{0 , 0 0 0}$} \\
\hline \multicolumn{6}{|l|}{ Escolaridade } \\
\hline Até 3 anos & 19,0 & 1,00 & & 1,00 & \\
\hline 4-7 anos & 25,9 & 1,36 & $0,85-2,18$ & 1,20 & $0,76-1,89$ \\
\hline 8-11 anos & 28,9 & 1,52 & $0,97-2,39$ & 1,43 & $0,90-2,24$ \\
\hline \multirow[t]{2}{*}{$\geq 12$ anos } & 33,1 & 1,74 & $1,10-2,78$ & 1,97 & $1,24-3,14$ \\
\hline & & $\mathrm{p}$ de tendência ${ }^{3}$ & $=0,120$ & $p$ de tendência ${ }^{3}$ & $=0,022$ \\
\hline
\end{tabular}

${ }^{1}$ Consumo abusivo de álcool definido como ingestão de álcool em dia típico de $30 \mathrm{~g}$ ou mais para homens e $24 \mathrm{~g}$ ou mais para mulheres. Porcentagens na amostra ponderada.

${ }^{2}$ Regressão de Poisson ajustada para variáveis apresentadas na tabela.

${ }^{3}$ Valor de p para teste de tendência linear.

${ }^{1}$ Alcohol abuse defined as daily consumption of at least 30 grams of alcohol for men and 24 grams for women. Weighted sample percentages.

${ }^{2}$ Poisson Regression controlling for variables presented in table.

${ }^{3}$ Trend test.

à menor escolaridade e não-inserção no mercado de trabalho no sexo masculino.

Os dados auto-referidos sobre a frequência de consumo de álcool, no sexo masculino, mostram que 52,4\% dos entrevistados relataram consumir bebida alcoólica em frequência de duas a três vezes por semana ou mais. Para o sexo feminino, esta frequência foi de 21,0\%. Estes dados são consistentes com a literatura ${ }^{9,18-23}$ quanto à maior frequência de consumo de bebida alcoólica entre os homens. Em inquérito nacional envolvendo as 107 maiores cidades do Brasil, Galduróz \& Carlini (2007) ${ }^{22}$, relataram uso na vida de álcool de $77,3 \%$ para os homens e $60,6 \%$ para as mulheres.

Em nosso estudo, o consumo abusivo de álcool em ambos os sexos foi inversamente associado à faixa etária, sendo a razão de prevalências ajustada de 0,73 (IC 95\%: 0,56-0,96) para os homens de 50-59 anos, e de 0,58 (IC 95\%: 0,35-0,95) para as mulheres na mesma faixa etária. Diversos estudos demonstram a associação inversa entre idade e consumo de álcool na população adulta, porém não de forma unívoca. Almeida \& Coutinho $(1993)^{18}$ observaram maior proporção de consumidores de álco- 
Tabela 5 - Dependência de álcool' (\%) segundo variáveis demográficas e socioeconômicas em população adulta do sexo masculino, Estado de São Paulo.

Table 5 - Alcohol dependence ${ }^{1}(\%)$ according to demographic and socioeconomic variables in male adults, State of São Paulo, Brazil

\begin{tabular}{|c|c|c|c|c|c|}
\hline \multirow[t]{2}{*}{ Variável } & \multirow[t]{2}{*}{$\%^{1}$} & \multicolumn{2}{|c|}{ Razão de Prevalências } & \multicolumn{2}{|c|}{ Razão de Prevalências } \\
\hline & & Bruta & IC 95\% & Ajustada $^{2}$ & IC 95\% \\
\hline \multicolumn{6}{|l|}{ Escolaridade } \\
\hline$<3$ anos & 61,1 & 1,00 & & 1,00 & \\
\hline 4-7 anos & 57,1 & 0,93 & $0,59-1,47$ & 1,08 & $0,64-1,84$ \\
\hline 8-11 anos & 41,5 & 0,67 & $0,36-1,24$ & 0,74 & $0,39-1,39$ \\
\hline \multirow[t]{2}{*}{$\geq 12$ anos } & 16,6 & 0,27 & $0,09-0,79$ & 0,32 & $0,10-1,01$ \\
\hline & & \multicolumn{2}{|c|}{$p$ de tendência ${ }^{3}=0,005$} & \multicolumn{2}{|c|}{$p$ de tendência ${ }^{3}=\mathbf{0 , 0 2 1}$} \\
\hline
\end{tabular}

Exerce atividade de trabalho

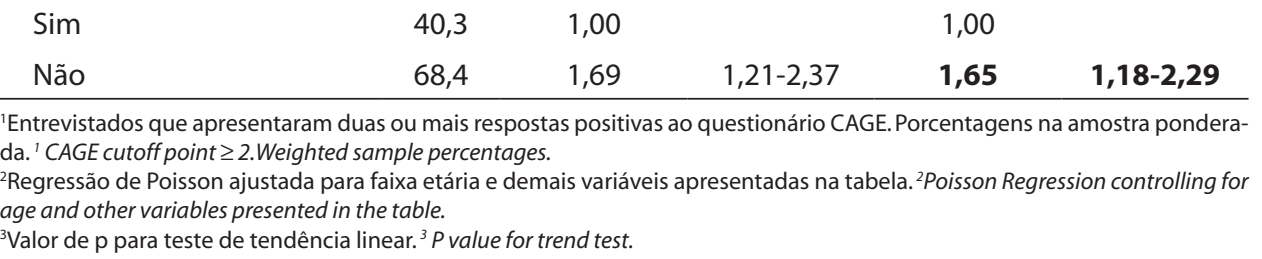

ol entre homens de 30 a 49 anos de idade, em moradores da Ilha do Governador, na cidade do Rio de Janeiro. Dados de 1999 coletados em 24 cidades do Estado de São Paulo apontam frequência de $70,5 \%$ de homens consumidores de álcool na faixa etária de 35 anos ou mais9. Em revisão de estudos publicados no Brasil entre $1943 \mathrm{e}$ 1985, também se observou concentração de consumo de álcool nos adultos jovens entre 20 e 49 anos de idade ${ }^{24}$.

Na população adulta do ISA-SP houve associação direta do consumo abusivo de álcool com o tabagismo e a escolaridade, em ambos os sexos. Diversos trabalhos corroboram a associação tabagismo-etilismo ${ }^{25-27}$. Alguns estudos evidenciam que o consumo de bebida alcoólica se distribui em todas as classes sociais ${ }^{18,23}$; entretanto, a maioria aponta para maior frequência de consumo em populações de menor renda e escolaridade $^{26-28}$. Outros fatores associados incluem: ser negro ou hispânico - nos Estados Unidos da América ${ }^{19}$ - migração e aculturação ${ }^{29}$, ser solteiro e fumar ${ }^{26}$.

Quanto à dependência de álcool, avaliada pelo teste CAGE, foram observadas duas ou mais respostas positivas em $14,8 \%$ dos homens e em $5,4 \%$ das mulheres que relataram consumir álcool. Isto corresponde a uma prevalência populacional de dependência de 10,4\% (IC 95\% 7,7-13,9) nos homens e 2,6\% (IC 95\% 1,6-4,4) nas mulheres. Estes resultados são semelhantes aos encontrados em outros estudos de dependência alcoólica realizados na população brasileira. Saalfeld \& Silva ${ }^{30}$ (1993) detectaram $6,3 \%$ de dependência etílica em serviço de cuidado primário a saúde, numa proporção de 18 homens para uma mulher. Rego et al. ${ }^{31}$ (1991), a partir de dados de inquérito domiciliar no município de São Paulo, encontraram CAGE positivo em $12,6 \%$ dos homens e $3,4 \%$ das mulheres. Em 1999, Galduroz et al. ${ }^{9}$ (2003) observaram $12,3 \%$ de dependência alcoólica no sexo masculino para a faixa etária 25-34 anos, e 8,9\% para os homens com idade igual ou superior a 35 anos. Para o sexo feminino, estes autores observaram dependência de $3,3 \%$ e $1,3 \%$, respectivamente. Inquérito nacional nas 107 cidades do Brasil com mais de 200.000 habitantes evidenciou prevalência de dependência alcoólica mais elevada que em nosso estudo, de $17,1 \%$ para o sexo masculino e $5,7 \%$ no sexo feminino ${ }^{22}$. 
No sexo masculino, observou-se associação inversa de CAGE positivo com escolaridade e com exercer atividade de trabalho. Os homens que relataram não exercer atividade remunerada de trabalho apresentaram razão de prevalências $65,0 \%$ maior em relação àqueles que estavam exercendo atividade de trabalho. Maior consumo de álcool e dependência em desempregados e indivíduos de menor escolaridade são observações relatadas por outros autores ${ }^{26,28,32,33}$.

Na avaliação do sexo feminino no ISA-SP, o CAGE positivo não se associou a nenhuma das variáveis sociodemográficas estudadas. Isto talvez reflita a natureza multifatorial mais complexa relacionada ao consumo e dependência de álcool no sexo feminino ${ }^{34}$. Nas mulheres, alguns autores relatam maior consumo abusivo de álcool e dependência nas faixas etárias mais jovens ${ }^{9,23}$. Em estudo de coorte de acompanhamento de gêmeas americanas, frequentar universidade, mesmo após controle para variáveis demográficas, socioeconômicas, de estilo de vida e de fatores familiares e ambientais, associou-se ao consumo ocasional de grandes quantidades de álcool ${ }^{35}$. No estudo de Galduroz et al. ${ }^{9}$ (2003), abrangendo 24 municípios do Estado de São Paulo, a prevalência de CAGE positivo foi de $4,3 \%$ nas mulheres de 18 a 24 anos. Caiu então para 3,3\% nas mulheres de 25 a 34 anos e para 1,3\% naquelas com idade maior ou igual a 35 anos.

Em nosso estudo, os achados que associam o consumo abusivo de álcool às mulheres mais jovens e de maior escolaridade, propõem reflexões sobre o papel social deste consumo e suas representações para este grupo específico.

A mensuração do consumo abusivo e dependência de álcool é controversa. Uma importante limitação de nossos dados foi não termos avaliado especificamente o consumo ocasional de grandes quantidades de álcool (binge drinking). Entretanto, a mensuração de nossos dados, a partir da quantidade e do tipo de bebidas alcoólicas ingeridas num dia típico de consumo de álcool, fornece estimativas muito mais fide- dignas das prevalências de consumo abusivo. Apesar de diversos estudos avaliarem o consumo de álcool apenas a partir de relatos de frequência de ingestão, sabe-se que esta estratégia pode subestimar a prevalência do consumo abusivo $^{36,37}$.

Os dados sobre etilismo apresentam grande variação quanto às medidas de consumo, forma de coleta e instrumentos utilizados. Existem importantes variações quanto à quantidade de álcool contida em cada unidade de bebida alcoólica ${ }^{38}$, o que diminui a precisão da avaliação da quantidade de álcool consumido. Outras limitações incluem a inexistência de um marcador biológico fidedigno e a multiplicidade de questionários utilizados. Mas, apesar destas questões, a avaliação do consumo de álcool a partir de informações auto-referidas já está validada como ótima estratégia para uso em estudos populacionais ${ }^{39}$.

O uso do teste CAGE apresenta algumas limitações. A validade do teste depende do contexto em que ele é utilizado. Em estudo de metanálise para avaliação do desempenho do questionário CAGE observou-se melhor desempenho em pacientes internados do que nos pacientes de atendimento primário ou ambulatoriais ${ }^{40}$. O CAGE tem sido aplicado em diversos contextos, como local de trabalho ${ }^{41}$, salas de emergência ${ }^{42,43}$ e em pacientes infectados pelo $\mathrm{HIV}^{44}$, e é considerado válido por alguns autores ${ }^{45}$ e não válido por outros ${ }^{46}$. Quanto ao uso populacional, Cherpitel ${ }^{47}$ (1998) concluiu que o CAGE não apresenta o mesmo desempenho quando aplicado à população em geral, comparando-se com a sua aplicação em determinadas situações clínicas. Outro estudo, comparando três diferentes instrumentos de diagnóstico de abuso/dependência de álcool, concluiu pelo melhor desempenho dos outros dois instrumentos em relação ao $\mathrm{CAGE}^{48}$.

O resultado do teste CAGE ainda pode ser alterado pela ordem de apresentação das questões. Etter ${ }^{49}$ (2004) encontrou menor percentual de respostas positivas às perguntas do CAGE quando estas eram antecedidas por perguntas sobre quantidade 
e frequência de uso de álcool. No projeto ISA-SP, essas questões foram formuladas posteriormente a aplicação do CAGE.

Apesar das evidências consistentes sobre o efeito benéfico do uso moderado de álcool sobre as doenças cardiovasculares ${ }^{50-52}$, e do seu possível efeito sobre a redução da mortalidade geral ${ }^{53}$, as conseqüências danosas são muitas e parecem estar mais relacionadas ao consumo abusivo ocasional ${ }^{54}$. É preocupante a frequência elevada de consumo abusivo observada em nosso estudo, principalmente entre os homens, sendo que $61,1 \%$ dos etilistas do sexo masculino com até três anos de escolaridade apresentaram CAGE positivo.

Estudos de tendência nos Estados
Unidos da América apontam uma estagnação no declínio do consumo de bebidas alcoólicas ${ }^{25,55}$. Por ser socialmente aceito, e também pela alta prevalência de consumidores e dependentes, é essencial a identificação dos segmentos sociodemográficos mais vulneráveis ao consumo abusivo e dependência de álcool. Novamente, as associações reveladas em nosso estudo entre a dependência/abuso e não estar exercendo atividade remunerada de trabalho, no sexo masculino, e a maior prevalência em mulheres de escolaridade universitária, sugerem componentes para programas de intervenção e controle.

Conflitos de interesse: não há.

\section{Referências}

1. World Health Organization. WHO Global Status Report on Alcohol. Department of Mental Health and Substance Abuse. Geneva: World Health Organization; 2004.

2. Paljärvi T, Mäkelä P, Poikolainen K. Pattern of drinking and fatal injury: a population-based follow-up study of Finnish men. Addiction 2005; 100: 1851-9.

3. Salomé H, French M, Matzger H, Weisner C. Alcohol consumption, risk of injury, and high-cost medical care. J Behav Health Serv Res 32: 368-80.

4. Cunradi C, Caetano R, Schafer J. Alcohol-related problems, drug use, and male intimate partner violence severity among US couples. Alcohol Clin Exp Res 2002; 26: 493-500.

5. Mukamal K, Mittleman M, Longstreth WJ, Newman A, Fried L, Siscovick D. Self-reported alcohol consumption and falls in older adults: cross-sectional and longitudinal analyses of the cardiovascular health study. J Am Geriatr Soc 2004; 52: 1174-9.

6. Polednak A. Recent trends in incidence rates for selected alcohol-related cancers in the United States. Alcohol Alcohol 40: 234-8.

7. Cook R, Clark D. Is there an association between alcohol consumption and sexually transmitted diseases? A systematic review. Sex Transm Dis 2005; 32: 156-64.

8. Ramstedt M. Alcohol consumption and liver cirrhosis mortality with and without mention of alcohol--the case of Canada. Addiction 2003; 98: 1267-76.

9. Galduróz J, Noto A, Nappo S, Carlini E. First household survey on drug abuse in São Paulo, Brazil, 1999: principal findings. Sao Paulo Med J 2003; 121: 231-7.
10. Galduroz J, Noto A, Nappo S, Carlini, EA. Comparações dos resultados de dois levantamentos domiciliares sobre o uso de drogas psicotrópicas no Estado de São Paulo nos anos de 1999 e 2001. J Bras de Psiquiatr 2003; 52: 4351.

11. Mokdad A, Marks J, Stroup D, Gerberding J. Actual causes of death in the United States, 2000. JAMA 2004; 291: 1238-45.

12. Moreira L, Fuchs F, Moraes R, Bredemeier M, Cardozo $\mathrm{S}$, Fuchs $\mathrm{S}$ et al. Alcoholic beverage consumption and associated factors in Porto Alegre, a southern Brazilian city: a population-based survey. J Stud Alcohol 1996; 57: 253-9.

13. Masur J, Monteiro M. Validation of the "CAGE" alcoholism screening test in a Brazilian psychiatric inpatient hospital setting. Braz J Med Biol Res 1983; 16: 215-8.

14. Skov T, Deddens J, Petersen M, Endahl L. Prevalence proportion ratios: estimation and hypothesis testing. Int J Epidemiol 1998; 27: 91-5.

15. Barros A, Hirakata V. Alternatives for logistic regression in cross-sectional studies: an empirical comparison of models that directly estimate the prevalence ratio. $B M C$ Med Res Methodol 2003; 3: 21.

16. Francisco P, Donalisio M, Barros M, Cesar C, Carandina L, Goldbaum M. Association measures in cross-sectional studies with complex samplings: odds ratio and prevalence ratio. Rev Bras Epidemiol 2008; 11: 347-55.

17. Casswell S, Huckle T, Pledger M. Survey data need not underestimate alcohol consumption. Alcohol Clin Exp Res 2002; 26: 1561-7. 
18. Almeida L, Coutinho ES. Prevalence of alcoholic beverage consumption and alcoholism in a metropolitan region of Brazil. Rev Saude Publica 1993; 27: $23-9$.

19. Galduróz J, Caetano R. Epidemiology of alcohol use in Brazil. Rev Bras Psiquiatr 2004; 26(S1): 3-6.

20. Galduróz J, Noto A, Nappo S, Carlini E. Trends in drug use among students in Brazil: analysis of four surveys in 1987, 1989, 1993 and 1997. Braz J Med Biol Res 2004; 37: 523-31.

21. Galduróz J, Noto A, Nappo S, Carlini E. Household survey on drug abuse in Brazil: study involving the 107 major cities of the country--2001. Addict Behav 2005; 30: 545-56.

22. Galduróz J, Carlini E. Use of alcohol among the inhabitants of the 107 largest cities in Brazil--2001. Braz J Med Biol Res 2007; 40: 367-75.

23. Costa J, Silveira M, Gazalle F, Oliveira S, Hallal P, Menezes A et al. Heavy alcohol consumption and associated factors: a population-based study. Rev Saude Publica 2004; 38: 284-91.

24. Cardim M, Assis S, M S, Iguchi T, Morgado A. Epidemiologia descritiva do alcoolismo em grupos populacionais do Brasil. Cad Saúde Pública 1986; 2: 191211.

25. Kerr W, Greenfield T, Bond J, YeY, Rehm J. Age-periodcohort modelling of alcohol volume and heavy drinking days in the US National Alcohol Surveys: divergence in younger and older adult trends. Addiction 2009; 104: 27-37.

26. Karlamangla A, Zhou K, Reuben D, Greendale G, Moore A. Longitudinal trajectories of heavy drinking in adults in the United States of America. Addiction 2006; 101: 91-9.

27. Chaieb J, Castellarin C. Smoking associated with alcoholism: introduction to the major human dependencies. Rev Saude Publica 1998; 32: 246-54.

28. Casswell S, Pledger M, Hooper R. Socioeconomic status and drinking patterns in young adults. Addiction 2003; 98: 601-10.

29. Zemore S. Re-examining whether and why acculturation relates to drinking outcomes in a rigorous, national survey of latinos. Alcohol Clin Exp Res 2005; 29: 2144-53.

30. Saalfeld V, Silva M. Prevalência do alcoolismo em cuidado primário à saúde. Pesqui Méd (Porto Alegre) $1993 ; 27: 5-9$.

31. Rego R, Berardo F, Rodrigues S, Oliveira Z, Oliveira $\mathrm{M}$, Vasconcellos $\mathrm{C}$ et al. Risk factors for chronic noncommunicable diseases: a domiciliary survey in the municipality of São Paulo, SP (Brazil). Methodology and preliminary results. Rev Saude Publica 1990; 24: 277-85.

32. Jhingan H, Shyangwa P, Sharma A, Prasad K, Khandelwal S. Prevalence of alcohol dependence in a town in Nepal as assessed by the CAGE questionnaire. Addiction 2003; 98: $339-43$.
33. Malyutina S, Bobak M, Kurilovitch S, Nikitin Y, Marmot $M$. Trends in alcohol intake by education and marital status in urban population in Russia between the mid 1980s and the mid 1990s. Alcohol Alcohol; 39: 64-9.

34. Nayak M, Kaskutas L. Risky drinking and alcohol use patterns in a national sample of women of childbearing age. Addiction 2004; 99: 1393-402.

35. Slutske W, Hunt-Carter E, Nabors-Oberg R, Sher K, Bucholz K, Madden P et al. Do college students drink more than their non-college-attending peers? Evidence from a population-based longitudinal female twin study. J Abnorm Psychol 2004; 113: 530-40.

36. Rehm J. Measuring quantity, frequency, and volume of drinking. Alcohol Clin Exp Res 1998; 22(S): 4-14.

37. Rehm J, Gmel G. Patterns of alcohol consumption and social consequences. Results from an 8-year follow-up study in Switzerland. Addiction 1999; 94: 899-912.

38. Kerr W, Greenfield T, Tujague J, Brown S. A drink is a drink? Variation in the amount of alcohol contained in beer, wine and spirits drinks in a US methodological sample. Alcohol Clin Exp Res 2005; 29: 2015-21.

39. Del Boca F, Darkes J. The validity of self-reports of alcohol consumption: state of the science and challenges for research. Addiction 2003; 98(S2): 1-12.

40. Aertgeerts B, Buntinx F, Kester A. The value of the CAGE in screening for alcohol abuse and alcohol dependence in general clinical populations: a diagnostic metaanalysis. J Clin Epidemiol 2004; 57: 30-9.

41. Amaral R, Malbergiera A. Evaluation of a screening test for alcohol-related problems (CAGE) among employees of the Campus of the University of São Paulo. Rev Bras Psiquiatr 2004; 26: 156-63.

42. Soderstrom C, Smith G, Kufera J, Dischinger P, Hebel J, McDuff D, Gorelick D et al. The accuracy of the CAGE, the Brief Michigan Alcoholism Screening Test, and the Alcohol Use Disorders Identification Test in screening trauma center patients for alcoholism. J Trauma 1997; 43: 962-9.

43. Cherpitel C, Borges G. A comparison of substance use and injury among Mexican American emergency room patients in the United States and Mexicans in Mexico. Alcohol Clin Exp Res 2001; 25: 1174-80.

44. Samet J, Phillips S, Horton N, Traphagen E, Freedberg K. Detecting alcohol problems in HIV-infected patients: use of the CAGE questionnaire. AIDS Res Hum Retroviruses 2004; 20: 151-5.

45. Saremi A, Hanson R, Williams D, Roumain J, Robin R, Long $\mathrm{J}$ et al. Validity of the CAGE questionnaire in an American Indian population. J Stud Alcohol 2001; 62: 294-300.

46. Bisson J, Nadeau L, Demers A. The validity of the CAGE scale to screen for heavy drinking and drinking problems in a general population survey. Addiction 1999; 94: 715-22. 
47. Cherpitel C. Performance of screening instruments for identifying alcohol dependence in the general population, compared with clinical populations. Alcohol Clin Exp Res 1998; 22: 1399-404.

48. Cherpitel C. Screening for alcohol problems in the U.S. general population: comparison of the CAGE, RAPS4, and RAPS4-QF by gender, ethnicity, and service utilization. Rapid Alcohol Problems Screen. Alcohol Clin Exp Res 2002; 26: 1686-91.

49. Etter J. Asking about quantity and frequency of alcohol consumption before asking the CAGE questions produces lower ratings on the CAGE test. Drug Alcohol Depend 2004; 74: 211-4.

50. Wilkins K. Moderate alcohol consumption and heart disease. Health Rep 2002; 14: 9-24.

51. Standridge J, Zylstra R, Adams S. Alcohol consumption: an overview of benefits and risks. South Med J 2004; 97: 664-72.
52. Burger M, Mensink G, Brönstrup A, Thierfelder W, Pietrzik K. Alcohol consumption and its relation to cardiovascular risk factors in Germany. Eur J Clin Nutr 2004; 58: 605-14.

53. Doll R, Peto R, Boreham J, Sutherland I. Mortality in relation to alcohol consumption: a prospective study among male British doctors. Int J Epidemiol 2005; 34: 199-204.

54. Rehm J, Gmel G, Room R, Frick U. Average volume of alcohol consumption, drinking patterns and related burden of mortality in young people in established market economies of Europe. Eur Addict Res 2001; 7: 148-51.

55. Greenfield T, Midanik L, Rogers J. A 10-year national trend study of alcohol consumption, 1984-1995: is the period of declining drinking over? Am J Public Health 2000; 90: 47-52.

Recebido em: 21/10/09

Aprovado em: 30/03/10 